\title{
Influence of oxidative stress on inducing micturition dysfunction following chronic infravesical obstruction and the protective role of an antioxidant diet - association of in vivo and in vitro studies in rats
}

Sérgio Bisogni, Fabio Thadeu Ferreira, Arnaldo Amstalden Neto, Leandro Oliveira Chiarelli, Valdemar Ortiz

Geriatric Urology Service, Federal University of São Paulo (UNIFESP), SP, Brazil.

\section{ABSTRACT}

Objective: This study was developed to determine whether the generation of free radicals, induced by ischemia followed by reperfusion in a model of chronic intravesical obstruction in rats, would lead to damage in the detrusor. It also investigates the possible protective action of the flavonoid galangin on the tissue lesion induced by lipid peroxidation.

Materials and Methods: Twenty-one male rats were divided into three groups of seven animals each. Group A was subjected to a sham procedure; group B to partial obstruction of the bladder neck; and group $\mathrm{C}$ to partial obstruction of the bladder neck, but also received a diet rich in the flavonoid galangin. All the animals were subjected to urodynamic evaluation and then sacrificed. The bladders were sent for enzymatic tests. Results: The urodynamic showed that group B developed significantly greater numbers of involuntary contractions of the detrusor, greater post-micturition residue and lower compliance.

The group A presented TEAC levels greater than to the group B. Comparative analysis of group A, B and C demonstrated significantly greater malondialdehyde levels in group B in relation to groups $\mathrm{A}$ and $\mathrm{C}$. The group B presented smaller contraction amplitudes than did groups A and C, in electrically stimulated contractions.

Conclusions: That oxidative stress is implicated in the damage to the detrusor musculature following a period of chronic intravesical obstruction. We show, for the first time, that administration of an antioxidant prior to and following the start of chronic obstruction makes it possible to avoid the cellular lesions that cause detrusor dysfunction.

\section{ARTICLE INFO}

\section{Key words:}

Urinary Bladder; Oxidative stress; Muscle Contraction; Flavonoids

Int Braz J Urol. 2012; 38: 552-60

Submitted for publication: May 30, 2011

Accepted after revision: January 12, 2012

\section{INTRODUCTION}

Overactive Bladder Syndrome (OBS) is a complex of symptoms that include micturitional urgency with or without urge incontinence, frequency and nocturia (1), without any demonstrable anatomical cause. The International
Continence Society regards OBS as a syndrome for which the physiopathology involved is not precisely known (1).

OBS is a recently defined entity. Thus, its prevalence and natural history have not been well studied. In our setting, Telöken et al. (2) conducted a population survey involving 848 
people aged between 15 and 55 years, and 18,9\% reported having symptoms compatible with OBS.

The symptoms of OBS have many causes, and a variety of factors may contribute towards the pathogenesis of the disease (3).

Recently, experimental studies have demonstrated a positive association between the triggering of OBS and the generation of free radicals $(4,5)$. The results from an experimental study involving smooth muscle fragments subjected to periods of repeated electrical stimulation demonstrated high levels of lipid peroxidation products, and decreased smooth muscle contractility of the bladder, proportional to the level of oxidative stress (4). Masick et al. (5) demonstrated the existence of deterioration in muscle contraction, and also the emergence of oxygen-reactive species, in the bladders of animals subjected to chronic urethral obstruction. These authors suggested that the formation of free radicals was an important factor in decreasing the detrusor contractility observed in patients with benign prostatic hyperplasia.

Over recent years, there has been great interest in developing new therapeutic methods for treating HBP. The main objective is to develop drugs that selectively inhibit the involuntary contractions of the detrusor, thereby diminishing the symptoms without affecting micturition contraction.

Antioxidants protect the organism against free radicals and are important agents for maintaining an adequate state of health. Flavonoids are an important source of antioxidants. Among the more than 4000 different types of flavonoids, galangin is a flavonol that has important biological activities at low concentrations and has a low toxicity index, which makes it a flavonoid with properties of interest for clinical studies regarding its biological benefits.

By utilizing an in vivo model, the present authors have investigated the action of oxidative stress on the bladder musculature and the possible protective action from the use of antioxidants. Thus, the principal objective of this study was to determine whether the oxidative stress induced by ischemia followed by reperfusion, in a model of chronic infravesical obstruction in rats, would lead to damage of the detrusor musculature.

\section{MATERIALD AND METHODS}

\section{Arrangement of the groups}

Twenty-one immature male rats weighing between 250 and 300 grams and aged 6-8 weeks were utilized in the experiment. These animals were divided into three groups of seven animals each (the sample size was determined statistically). They were kept under adequate conditions of hygiene in a climate-controlled vivarium and received controlled feed.

Group A was submitted to a sham procedure consisting of opening and closing the abdominal wall without positioning an obstructive ring on the bladder neck. Four weeks after the procedure, the animals were submitted to urodynamic evaluation and then sacrificed to remove the bladder.

Group B was submitted to a surgical procedure to create partial obstruction of the bladder neck (as described below). Four weeks after the procedure, the animals were submitted to urodynamic evaluation and then sacrificed to remove the bladder.

Group C was submitted to a surgical procedure to create partial obstruction of the bladder neck (as described below). Four weeks before the procedure, an antioxidant-rich diet rich was introduced (containing the flavonoid galangin). After the procedure, the diet was maintained for four weeks afterwards, when the animals were submitted to urodynamic evaluation and then sacrificed to remove the bladder.

\section{Antioxidant diet}

Group C received a normal purine diet, like the other groups, but with the addition of a high proportion of the flavonoid galangin (30 $\mu \mathrm{M} /$ day). The diet was devised by the chemistry laboratory of the institution at which the study was conducted.

The addition of galangin did not alter the color, texture, smell or flavor of the diet.

The animals' daily intake of this diet was controlled so that all of them received $40 \mathrm{~g}$ of feed/day. 
Description of the procedure for creating the obstruction

Under anesthesia using xylazine $(0.87 \mathrm{mg} /$ $\mathrm{kg}$ intraperitoneally) and ketamine $(43.3 \mathrm{mg} / \mathrm{kg}$ intraperitoneally), the abdominal cavity was opened up in layers and the periurethral space was delicately dissected. A silver ring of $2 \mathrm{~mm}$ in diameter was positioned around the bladder neck. This model made possible the institution of a gradual obstruction process parallel to the animal's growth, similar to a model for urological diseases that cause infravesical obstruction (6).

\section{Urodynamic evaluation}

Under anesthesia using urethane $(1 \mathrm{mg} / \mathrm{Kg}$ intraperitoneally) (the drug of choice for urodynamic evaluations in animals, that enable micturitional assessment), two children's angiographic catheters were inserted percutaneously into the bladder (24-gauge, 1-2 cm, Baxter Healthcare AS). One of the catheters was connected to an infusion pump with a capacity of $0.23 \mathrm{~mL} / \mathrm{min}$ and the other to a pressure transducer. The volume urinated was collected using a graduated pipette connected to the pressure transducer (Baxter) in order to measure the volume urinated. Ten micturition cycles were evaluated and the following parameters were observed: presence and frequency of involuntary contractions of the detrusor during bladder filling (contractions for which the maximum amplitude reached one-third of the maximum detrusor pressure during micturition), maximum detrusor pressure, compliance, residual volume, micturition volume and bladder capacity.

Following the urodynamic evaluation, the animals were sacrificed and the bladder was removed at the level of the bladder neck and processed for enzymatic and electrophysiological tests. After removing the bladder, it was sectioned longitudinally between the ureteral openings: the left half was sent for electrophysiological tests and the right half for enzymatic tests.

\section{Tissue preparation for enzymatic tests}

The right half of the bladder, composed of smooth muscle and mucosa, was exposed to liquid nitrogen, weighed, dispersed in 1.15\% KCL and homogenized to a concentration of $50 \mathrm{mg} / \mathrm{mL}$ for 10s, using a polytron. The mitochondria and microsomes were then isolated through different centrifugation runs, as described previously (7).

\section{Malondialdehyde (MDA)}

Samples of bladder tissue (processed previously, as described above) were placed in glass tubes, and 1.15\% 0.05M KCL and $1 \mathrm{mM} \mathrm{FeSO} 4$ were introduced into $500 \mathrm{~mL}$ of tissue extract. The samples were incubated at $37^{\circ} \mathrm{C}$ for $60 \mathrm{~min}$. At the end of the incubation, the reaction was halted by means of adding $150 \mathrm{~mL}$ of $40 \%$ trichloroacetic acid. The material was put into tubes and centrifuged at $1000 \times \mathrm{x}$ for $1 \mathrm{~min}$. After centrifuging, 100 $\mathrm{mL}$ of the supernatant was incubated with $750 \mathrm{~mL}$ of perchloric acid (PCA) and thiobarbituric acid (TBA) $(2.3 \%$ PCA, $0.53 \%$ TBA, $\mathrm{pH} 7.4)$ at $100^{\circ} \mathrm{C}$ for $60 \mathrm{~min}$ and then in ice for $10 \mathrm{~min}$. To each tube, $2 \mathrm{~mL}$ of 1-butanol was added, and the tubes were inverted for $2 \mathrm{~min}$. The tubes were centrifuged at $1000 \times \mathrm{G}$ for $5 \mathrm{~min}$ and the top layer was measured fluorometrically at $532 \mathrm{~nm}$. Total quantification of proteins was done by means of the Micro BCA protein analyzer, and the results were expressed as nmoL MDA/mg protein (7).

The MDA levels were described in relation to the total protein levels.

\section{Total antioxidant capacity - trolox (TEAC)}

To measure the total antioxidant capacity, a previously standardized technique was utilized $(8,9)$. In short, the total antioxidant capacity was analyzed on the basis of the ability of a compound to eliminate the ABTS radical in 6 min. ABTS was produced the reaction between $7 \mathrm{mM}$ ABTS and $2.45 \mathrm{mM}$ of potassium persulfate in water. This solution was stored in a darkened environment for 12-16 $\mathrm{h}$ before use. The extinction coefficient of ABTS at $734 \mathrm{~nm}$ is $1.5 \times 104 / \mathrm{moL} / \mathrm{cm}$.

The solution containing ABTS was diluted with phosphate (PBS) at $\mathrm{pH} 7.4$, until final absorption of $0.70 \pm 0.02$ at $734 \mathrm{~nm}$ was achieved, at $37^{\circ}$ C. Ten microliters of an antioxidant were added to $990 \mu \mathrm{L}$ of ABTS and the absorption at $734 \mathrm{~nm}$ was evaluated. This result was compared with a reaction without antioxidant, in which $10 \mu \mathrm{L}$ of the solvent was added to $990 \mu \mathrm{L}$ of ABTS. The reduction in absorption after adding the antioxidant 
was determined. The TEAC of the bladder tissue from the animals was calculated by adding the supernatant from the tissue to the ABTS solution. The tissue reacted instantly with the ABTS and the reaction was complete within a few seconds. The decrease in absorption at $734 \mathrm{~nm}$ was monitored over this period.

The TEAC levels were described in relation to the total protein levels.

\section{Preparation of tissue for physiological evaluation}

Fragments of $2 \times 2 \mathrm{~cm}$, consisting of the mucous layer, submucous fat and conjunctive tissue, were removed from the dorsum of the bladder cap with the aid of a binocular microscope. Similar-sized fragments of longitudinal smooth muscle, of $2 \mathrm{~mm}$ in diameter and $5 \mathrm{~mm}$ in length, were extracted for performing experiments. Only one fragment was taken from each animal, so as to utilize the greatest number of animals possible.

\section{Electrophysiological evaluation}

Organ bath: Each fragment was positioned vertically in an organ bath containing $5 \mathrm{~mL}$ of a standard nutrient solution (Krebs solution: 118 $\mathrm{mM} \mathrm{NaCl}, 5.6 \mathrm{mM} \mathrm{KCl}, 25 \mathrm{mM} \mathrm{NaHCO} 3,1.3 \mathrm{mM}$ NaH2P04, $2.5 \mathrm{mM} \mathrm{CaCl} 2,1.2 \mathrm{mM} \mathrm{MgSO} 4,6.1 \mathrm{mM}$ glycose; $\mathrm{pH} 7.4,95 \%$ 02, 5\% CO2), between two clips that were connected to a voltage transducer. An electrical field was generated (Hameg ${ }^{\circledR}$ Instruments) between two platinum electrodes. The input and output flow of bath solution was controlled by a system positioned at the bottom of the organ bath. The temperature was kept at $37^{\circ} \mathrm{C}$ by using a water heating system.

Protocol: The muscle fragments were individually inserted into the organ bath and incubated in the Krebs solution. Just after positioning the fragment in the organ bath, the "L0" point representing the length associated with maximum isometric strength was determined at a temperature of $37^{\circ} \mathrm{C}$. After determining Lo, the fragments were left to rest for a 30-minute period and then were subjected to different stimuli: electrical (EFS), Ach $(10 \mu \mathrm{moL} / \mathrm{L})$ and ATP $(10 \mu \mathrm{moL} / \mathrm{L})$. An interval of 10 minutes was maintained between applying each stimulus, in order to avoid muscle fatigue.

\section{Statistical evaluation}

The results were expressed as mean \pm standard deviation, when appropriate. The differences in maximum strength and time constant between pre and post-treatment readings were evaluated using SPSS 8.0 for Windows, by means of student's t-test and ANOVA with Dunnet's post-hoc test. The significance level chosen was 95\% $(p<0.05)$. It was unnecessary to normalize the readings for maximum strength and time constant, since the fragments were similar in size to reduce the variability in maximum basal amplitude.

\section{RESULTS}

\section{Urodynamic evaluation}

Table-1 summarizes the urodynamic findings of the different groups. The results demonstrated that the obstructed group (group B) developed significantly greater number of involuntary contractions of the detrusor during the bladder filling phase $(p<0.05)$, and also diminished maximum micturition flow $(\mathrm{p}<0.05)$, increased post-micturition residue $(\mathrm{p}<0.05)$ and decreased compliance $(\mathrm{p}<0.05)$.

There was no significant difference between groups $A$ and $C(p>0.05)$.

\section{Total antioxidant capacity}

Tissue TEAC measurements were obtained from 40 samples obtained from the supernatant of each animal. The results are represented in Figure-1.

The animals in group A (sham procedure) presented statistically greater TEAC levels $\left(111.57 \pm 9.50 \mu \mathrm{M} / \mathrm{mg}^{*} \mathrm{~mL}-1\right)$ than did group B (infravesical obstruction without antioxidant-rich diet) $\left(50.02 \pm 9.80 \mu \mathrm{M} / \mathrm{mg}^{*} \mathrm{~mL}-1\right)$. Group C (obstructed plus antioxidant-rich diet) presented TEAC of $80.54 \pm 9.99 \mu \mathrm{M} / \mathrm{mg}^{*} \mathrm{~mL}-1$, which was statistically lower than group A and greater than group B.

The highest TEAC values (in group A) suggest that the bladders in these animals were exposed to the least oxidizing damage. In contrast, the lowest TEAC values (in group B) suggest that these animals had a low antioxidant capacity, i.e. the greatest oxidizing damage. 
Table 1 - Findings from the urodynamic evaluation performed four weeks after the sham procedure (group A), partial obstruction of the bladder neck using a silver ring (group B) and chronic obstruction together with the use of the flavonoid galangin (group C). The data are expressed as means.

\begin{tabular}{lcccc}
\hline & Group A & Group B & Group C & $p$ \\
\hline Presence of ICD & absent & present & absent & not applicable \\
Frequency of ICD/min & 0 & 4 & 1 & 0.01 B vs. A + C \\
MaxDP & 28.7 & 51.2 & 31.4 & 0.03 B vs. A + C \\
Compl & 1.32 & 0.08 & 1.3 & 0.03 B vs. A + C \\
Rvol & 0.3 & 2.1 & 0.4 & 0.04 B vs. A + C \\
Mvol & 2.7 & 0.4 & 2.3 & 0.01 B vs. A + C \\
MaxCC & 3.1 & 2.5 & 2.9 & 0.3 \\
\hline
\end{tabular}

ICD: involuntary contraction of detrusor; MaxDP: maximum detrusor pressure $\left(\mathrm{cmH}_{2} \mathrm{O}\right)$; Compl: bladder compliance $\left(\mathrm{mL} / \mathrm{cmH}_{2} 0\right)$; Rvol: residual volume (mL); Mvol: micturitional volume (mL); MaxCC: maximum cystometric capacity (mL).

Figure 1 - Mean TEAC values of the samples obtained from the rats, in the different groups.

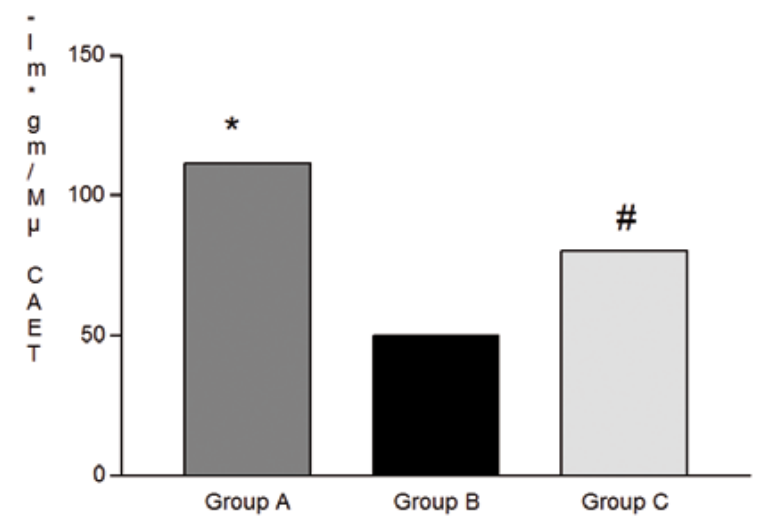

Group A: sham; Group B: obstructed; Group C: obstructed + galangin. ${ }^{*} p<0.05$ A vs. $B+C, \# p<0.05$ C vs. $A+B$.

\section{Malondialdehyde}

The MDA levels were expressed as $\mu \mathrm{M}$ $\mathrm{MDA} / \mathrm{mg}^{*} \mathrm{~mL}-1$ protein (Figure-2).

Since the MDA levels correlated negatively with the total antioxidant capacity, it can be assumed that the low MDA levels represented high antioxidant potential in the samples studied.
Figure 2 - Mean MDA values of the samples obtained from the rats, in the different groups.

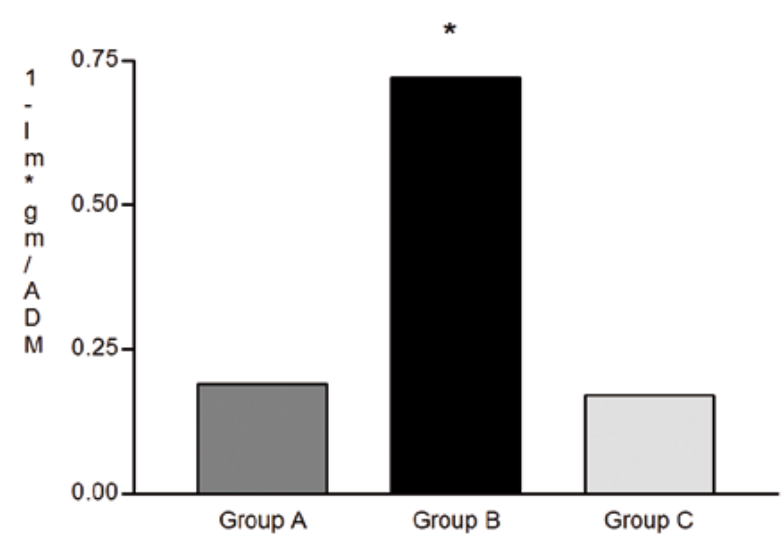

Group A: sham; Group B: obstructed; Group C: obstructed + galangin. ${ }^{*} p<0.05$ A vs. $B+C, \# p<0.05 C$ vs. $A+B$.

Comparative analysis of groups A (sham), B (obstructed) and C (obstructed + galangin) demonstrated that there were significantly greater MDA levels in group $\mathrm{B}$ than in groups $\mathrm{A}$ and $\mathrm{C}$ (respectively $0.19 \pm 0.069 ; 0.72 \pm 0.03$ and $0.17 \pm 0.04$ ).

In vitro evaluation - response of the detrusor musculature to electrical stimulation 
The data related to the detrusor response to different electrical frequencies are shown in Figure-3. At the frequencies of 16 and $34 \mathrm{~Hz}$, it was observed that the animals in the obstructed group (B) developed contractions of significantly lower amplitude $(\mathrm{p}<0.05)$ than did the sham group (A) and the obstructed group that received galangin (C).

In a preliminary study, it had been observed that the muscle contractions induced by electrical stimulation were totally suppressed by tetrodotoxin (10-6M), which is a nervous response inhibitor. Thus, the results obtained from the present study indicate that chronic obstruction leads to damage to the nervous response that is induced by the release of neurotransmitters at the presynaptic terminations.

In vitro evaluation- response of the detrusor musculature to acetylcholine

The data related to the detrusor response to acetylcholine are presented in Figure-4. The amplitudes of the muscle contraction induced by

Figure 3 - Response of the detrusor musculature to different electrical stimulation $(1,2,4,8,16$ and $32 \mathrm{~Hz})$.

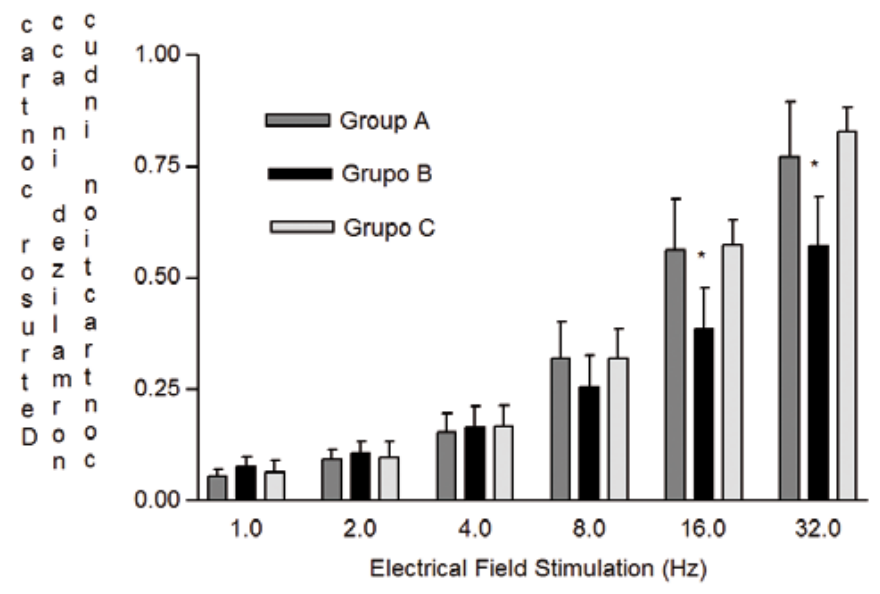

Group A: sham; Group B: obstructed; Group C: obstructed + galangin. ${ }^{*} p<0.05$.

Figure 4 - Response of the detrusor musculature to acetylcholine (Ach) $(10 \mu \mathrm{moL} / \mathrm{L})$.

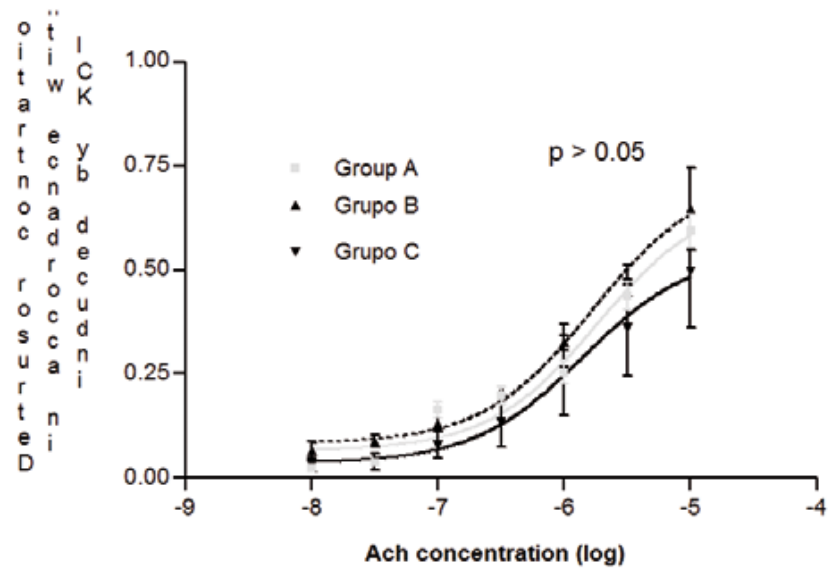

Group A: sham; Group B: obstructed; Group C: obstructed + galangin. 
acetylcholine in the different groups were statistically similar ( $p>0.05)$. Thus, it is suggested that the damage to the detrusor musculature induced by ischemia/reperfusion does not affect the muscarinic receptors.

In vitro evaluation - response of the detrusor musculature to ATP (adenosine triphosphate)

The data related to the detrusor response to ATP are shown in Figure-5. The amplitudes of the muscle contraction induced by ATP in the different groups were statistically similar ( $p>0.05)$. Thus, it is suggested that the damage to the detrusor musculature induced by ischemia/reperfusion does not affect the purinergic receptors.

Figure 5 - Response of the detrusor musculature to ATP (10 $\mu \mathrm{moL} / \mathrm{L})$.

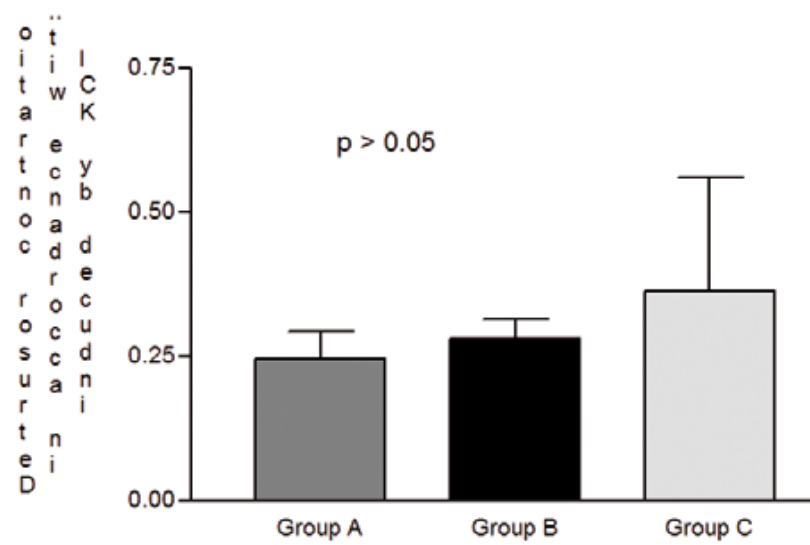

Group A: sham; Group B: obstructed; Group C: obstructed + galangin.

\section{DISCUSSION}

In this study, data from in vivo and in vitro evaluations were combined to assess detrusor function following a period of partial infravesical obstruction, and also the protective role of the flavonoid galangin on the detrusor response to chronic obstruction. Specifically, in vivo urodynamic evaluation data and in vitro isometric parameters and enzymatic tissue marker data were utilized to prove the hypothesis that the generation of free radicals has a role in detrusor damage after a period of chronic infravesical obstruction.
The possible role of oxidative stress in the physiopathology of bladder dysfunction has still not been elucidated, despite various studies conducted in this respect, in different centers around the world. Zhao et al. were the first investigators to suggest that a bladder submitted to acute distension would initially present a period of ischemia, followed by a period of reperfusion, which would lead to the generation of free radicals and tissue lesions (10). More recently, a study developed by Levin et al. demonstrated a possible relationship between a period of ischemia followed by reperfusion and the etiology of the bladder dysfunction subsequent to a period of chronic infravesical obstruction (7). These authors formulated the hypothesis that a state of oxidative stress would be present in a situation of chronic obstruction and that this would lead to the formation of metabolic end products that would cause damage to the phospholipids of the lipid membrane of the muscle cells. However, they did not find enough data to prove their hypothesis.

In the present investigation, two of the most widely used and accepted markers were utilized to demonstrate the existence of oxidative stress in the tissue (MDA and TEAC). MDA, which is the final product from the end product of lipid peroxidation induced by free radicals, has been implicated as the most important cause of destruction and damage in cell membranes. This occurs because the polyunsaturated fatty acids of cell membranes are degraded by this process, with consequent rupture of membrane integrity. Membrane peroxidation may lead to changes in membrane fluidity and permeability, and may also increase the protein degradation and cell lysis rates (11).

The total antioxidant capacity (TEAC), which is another marker used for indirectly determining the levels of oxidative stress in tissue, has been widely used for homogenized tissue from rats (12). This analysis assesses the antioxidant activity against the ABTS radical.

In the present study, the MDA concentration in the group subjected to the chronic obstruction procedure (B) was significantly greater than in the animals subjected to the sham procedure (A) and/or chronic obstruction with concomitant 
use of galangin (C). It was also observed that the total antioxidant capacity in group B was statistically lower than in the other two groups, which indicates the presence of significant oxidative damage in the samples studied. When taken together, these findings corroborate the hypothesis that high levels of lipid peroxidation are associated with chronic infravesical obstruction. We can suggest that the damage to the detrusor musculature induced by infravesical obstruction (proven via the low amplitudes of contractions induced by electric stimulation and the occurrence of bladder dysfunction during the urodynamic evaluation) is at least partially related to the formation of free radicals and cell membrane damage.

There is growing evidence that flavonoids have beneficial effects and are of special interest for preventing and treating specific diseases that are related to free radical generation (13). In the present study, the hypothesis tested was that one antioxidant (the flavonoid galangin) would have the capacity to avoid tissue damage induced by oxidative stress in the detrusor cells of guinea pigs. Galangin was chosen from among other flavonoids because it has been demonstrated to be one of the flavonoids with greatest relaxant power of smooth musculature (14). Furthermore, galangin is the most lipophilic of the flavonoids, which makes it appropriate for intravesical use due to the facility with which it penetrates the barrier formed by bladder mucosa (15).

The mechanism through which galangin protects the detrusor from tissue lesions following a period of chronic obstruction is a matter for speculation. Capasso \&t Tavares suggested in a study on rats in which they verified the action of this flavonoid on the amplitudes of bladder muscle contractions, that it would act on type L calcium channels (16). Levin et al. demonstrated that, when muscle tissue was exposed to free radical generation, there was an accumulation of calcium in the intracellular medium, which would be responsible for cell damage. Thus, they suggested that galangin would act through type L calcium channels, thereby avoiding increased transportation of calcium ions into cells (17). There would, of course, be a need for studies to elucidate the exact mechanism for the action of galangin, in or- der to characterize the diseases for which galangin could become part of the therapeutic arsenal.

In summary, the findings described in the present study allow the conclusion that oxidative stress has a role in the damage of the detrusor musculature resulting from a period of chronic infravesical obstruction. We also have, for the first time, shown that administration of an antioxidant prior to and following the beginning of chronic obstruction makes it possible to avoid the cellular lesions that cause detrusor dysfunction.

From a broader perspective, it is suggested that benign prostate hyperplasia (BPH) is associated with induced free radical formation. Free radicals would therefore be at least partially responsible for the different degrees and types of micturition dysfunction observed during the evolution of these patients. Together with this hypothesis, it is also suggested that the use of antioxidants would have a protective role against micturition dysfunction during the follow-up of patients with BPH.

\section{CONCLUSIONS}

These results allow the conclusion that oxidative stress is implicated in the damage of the detrusor musculature following a period of chronic infravesical obstruction. We also have, for the first time, shown that administration of an antioxidant prior to and following the beginning of chronic obstruction can avoid the cellular lesions that cause detrusor dysfunction.

\section{CONFLICT OF INTEREST}

None declared.

\section{REFERENCES}

1. Abrams P, Cardozo L, Fall M, Griffiths D, Rosier P, Ulmsten $U$, et al.: The standardisation of terminology of lower urinary tract function: report from the Standardisation Subcommittee of the International Continence Society. Neurourol Urodyn. 2002; 21: 167-78.

2. Teloken C, Caraver F, Weber FA, Teloken PE, Moraes JF, Sogari PR, et al.: Overactive bladder: prevalence and implications in Brazil. Eur Urol. 2006; 49: 1087-92. 
3. Ouslander JG: Management of overactive bladder. N Engl J Med. 2004; 350: 786-99.

4. Ohnishi N, Liu SP, Horan P, Levin RM: Effect of repetitive stimulation on the contractile response of rabbit urinary bladder subjected to in vitro hypoxia or in vitro ischemia followed by reoxygenation. Pharmacology. 1998; 57: 13947.

5. Masick JM, Levin RM, Hass MA: The effect of partial outlet obstruction on prostaglandin generation in the rabbit urinary bladder. Prostaglandins Other Lipid Mediat. 2001; 66: $211-9$

6. Mostwin JL, Karim OM, van Koeveringe G, Brooks EL: The guinea pig as a model of gradual urethral obstruction. $J$ Urol. 1991; 145: 854-8.

7. Levin RM, Leggett RE, Whitbeck C, Murakami T, Kambara T, Aikawa K: Oral Kohki Tea and its protective effect against in vitro ischemic damage to the bladder. Neurourol Urodyn. 2004; 23: 355-60.

8. Berg van den R, Haenen GRMM, van den Berg H, Bast A. Applicability of an improved Trolox equivalent antioxidant capacity (TEAC) assay for evaluation of antioxidant capacity measurements of mixtures. Food Chem. 1999; 66: 511-7.

9. Re R, Pellegrini N, Proteggente A, Pannala A, Yang M, RiceEvans C: Antioxidant activity applying an improved ABTS radical cation decolorization assay. Free Radic Biol Med. 1999; 2: $1231-7$.

10. Zhao Y, Levin SS, Wein AJ, Levin RM: Correlation of ischemia/reperfusion or partial outlet obstruction-induced spectrin proteolysis by calpain with contractile dysfunction in rabbit bladder. Urology. 1997; 49: 293-300.
11. García JJ, Reiter RJ, Guerrero JM, Escames G, Yu BP, Oh CS, et al.: Melatonin prevents changes in microsomal membrane fluidity during induced lipid peroxidation. FEBS Lett. 1997; 408: 297-300.

12. Weight SC, Bell PR, Nicholson ML: Renal ischaemia--reperfusion injury. Br J Surg. 1996; 83: 162-70.

13. Tokalov SV, Kind B, Wollenweber E, Gutzeit HO: Biological effects of epicuticular flavonoids from Primula denticulata on human leukemia cells. J Agric Food Chem. 2004; 52: 239-45.

14. Hammad HM, Abdalla SS: Pharmacological effects of selected flavonoids on rat isolated ileum: structure-activity relationship. Gen Pharmacol. 1997; 28: 767-71.

15. Heijnen CG, Haenen GR, Oostveen RM, Stalpers EM, Bast A: Protection of flavonoids against lipid peroxidation: the structure activity relationship revisited. Free Radic Res. 2002; 36: 575-81.

16. Capasso R, Tavares IA: Effect of the flavonoid galangin on urinary bladder rat contractility in-vitro. J Pharm Pharmacol. 2002; 54: 1147-50.

17. Levin RM, Leggett R, Whitbeck C, Horan P: Effect of calcium and calcium chelator on the response of the bladder to in vitro ischaemia. Br J Urol. 1998; 82: 882-7.

Correspondence address: Dr. Fabio Thadeu Ferreira Rua Duque de Caxias, 780 $10^{\circ}$ andar / 102-104. Campinas, SP, 13015-311, Brazil E-mail: fabio.urologia@gmail.com 